Cindrilla Chumduri und Margherita Yayoi Turco

\title{
3.7 Organoide des weiblichen Reproduktionstraktes ${ }^{34}$
}

Zusammengefasst und aus dem Englischen übersetzt von Lilian Marx-Stölting und Thomas Burgold

\section{Einleitung}

Der Reproduktionstrakt der Frau erfüllt verschiedene Funktionen, die in erster Linie die Geburt von Nachkommen ermöglichen und somit den Fortbestand der menschlichen Spezies sichern. Er reguliert nicht nur die Eizellreifung und bietet eine schützende Umgebung für die Befruchtung der Eizelle sowie die anschließende Einnistung (Implantation) des Embryos, sondern übernimmt auch dessen Ernährung und gewährleistet dadurch Wachstum und Entwicklung des Embryos. Nach der Geburt regeneriert sich der weibliche Reproduktionstrakt und ermöglicht so den Beginn einer erneuten Schwangerschaft.

Seit Kurzem sind Wissenschaftler in der Lage, Teile des komplexen weiblichen Reproduktionstraktes mithilfe dreidimensionaler organähnlicher Zellstrukturen, sogenannter Organoide, nachzubilden. Im Folgenden werden der aktuelle Forschungsstand und Anwendungspotenziale dieser vielversprechenden In-vitro-Modelle, sowie ihr möglicher Beitrag zur Verbesserung der reproduktiven Gesundheit von Frauen, diskutiert.

Der weibliche Reproduktionstrakt besteht aus verschiedenen Bereichen (siehe Abbildung 1): den Eierstöcken, in denen die Eizellen heranreifen und freigesetzt werden, den Eileitern, die die Eizelle nach dem Eisprung und der Befruchtung in die Gebärmutter (Uterus) transportieren, dem Uterus, in dem die Implantation des Embryos und die Schwangerschaft stattfinden, und dem Gebärmutterhals (Cervix), der den Uterus mit der Vagina verbindet und durch den die Samenzellen in den Uterus eindringen. Die Schleimhaut, die all diese verschiedenen Regionen umgibt, ermöglicht den Transport

34 Dieser Beitrag ist eine deutsche Zusammenfassung des Review-Artikels „Organoids of the Female Reproductive Tract" von Cindrilla Chumduri und Margherita Yayoi Turco, der 2020 im Journal of Molecular Medicine erschienen ist und eine umfangreiche Darstellung des wissenschaftlichen Sachstands sowie der aktuellen Literatur zum Thema (Stand: Frühjah 2020) bietet. 
von Ei- und Samenzellen und den erfolgreichen Transfer der befruchteten Eizelle in den Uterus. Zudem verhindert die Schleimhaut das Eindringen von Krankheitserregern.

Abbildung 1: Anatomie des menschlichen Reproduktionstraktes mit zwei Eierstöcken, zwei Eileitern, Uterus, Cervix und Vagina
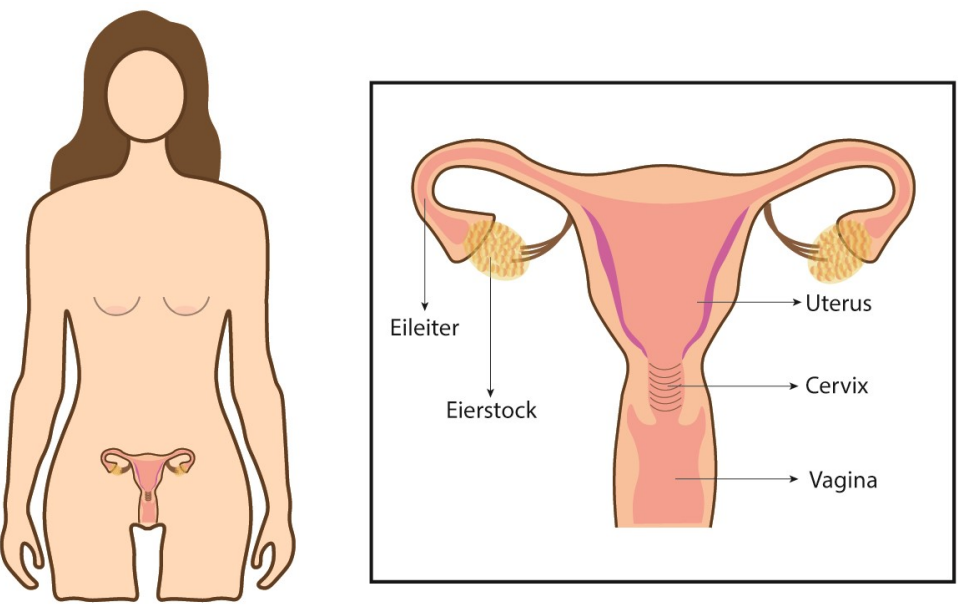

Die vielfältigen Funktionen des weiblichen Reproduktionstraktes während Menstruationszyklus, Schwangerschaft und Geburt hängen von einer ausgeglichenen und dynamischen Hormonregulation ab. Dabei steuern Hormone des Hypothalamus, der Hypophyse $^{35}$ und der Eierstöcke durch ihr Zusammenspiel die Wechselwirkungen zwischen Epithel-, Stroma- ${ }^{36}$ und Immunzellen. Störungen dieser Prozesse führen zu einem Zusammenbruch der Homöostase, also des dynamischen Gleichgewichtes innerhalb des weiblichen Reproduktionstraktes. Die Folgen können nicht nur reproduktives

35 Der Hypothalamus ist ein Teil des Zwischenhirns und zentrale Regulationsstelle zwischen dem endokrinen System (dem Hormonsystem) und dem Nervensystem. Die Hypophyse ist ein endokrines Organ im Nervensystem, d. h. es stellt Hormone her und schüttet diese aus.

36 Die Zellen des Epithelgewebes bedecken die inneren und äußeren Körperoberflächen (Oberflächenepithel) und sind funktioneller Bestandteil von sekretproduzierenden Drüsen (Drüsenepithel). Stromazellen bilden das Bindegewebe eines Organs und üben in diesem eine Stütz- und Ernährungsfunktion aus. 
Versagen sein, sondern auch eine Reihe von Krankheiten, darunter Endometriose ${ }^{37}$ und Karzinome (Krebs). Obwohl viele Frauen an diesen Erkrankungen leiden, ist das Wissen um ihre Entstehung noch begrenzt und es fehlen effiziente Behandlungsmöglichkeiten. Das zunehmende Alter von Müttern, ungünstige Ernährungsweisen und weitere Lebensstilveränderungen sowie verschiedene Umweltfaktoren (wie etwa Stoffe, die das Hormonsystem stören, sogenannte endokrine Disruptoren) führen zu einer Zunahme dieser Erkrankungen. Daher ist ein besseres Verständnis der zellulären und molekularen Mechanismen, die den Prozessen im weiblichen Reproduktionstrakt zugrunde liegen, von immer größerer Bedeutung.

Organoidsysteme stellen wichtige experimentelle Modelle dar, die zum einen die zelluläre Heterogenität und zum anderen die physiologischen, anatomischen und funktionellen Eigenschaften der Organe in vitro nachbilden können. Diese Organoidkulturen können entweder aus pluripotenten oder aus gewebespezifischen (adulten) Stammzellen gewonnen werden. ${ }^{38}$ Organoide aus adulten Stammzellen können dabei sowohl aus gesundem als auch aus erkranktem Gewebe gezüchtet werden. Dies ermöglicht nicht nur die Untersuchung von normalen physiologischen Vorgängen, sondern ebenso von gestörten krankheitsspezifischen Abläufen. Darüber hinaus können Organoidsysteme für die Medikamentenentwicklung und die personalisierte Medizin genutzt werden.

\subsubsection{Organoide als Werkzeuge zur Erforschung des weiblichen Reproduktionstraktes}

Vor der Entdeckung und Entwicklung von Organoiden wurde der weibliche Reproduktionstrakt typischerweise an aus Geweben isolierten Zellen (sogenannten Primärzellen), an von Karzinomen abgeleiteten Zelllinien ${ }^{39}$ oder direkt an entnommenen Geweben erforscht. Diese Methoden weisen jedoch zahlreiche Nachteile auf: Primärzellen haben in vitro nur eine kurze Lebensspanne und lassen sich daher nicht dauerhaft kultivieren. Die gebräuchlichen Zelllinien aus Karzinomen weisen zum einen, im Vergleich zu gesunden Zellen, eine veränderte Chromosomenanzahl sowie andere

37 Eine Endometriose liegt vor, wenn gebärmutterschleimhautartige Zellen außerhalb der Gebärmutter wachsen.

38 Zur Beschreibung und Gewinnung von Organoiden aus verschiedenen Stammzelltypen siehe Einleitung, Kap. 2.1.

39 Eine Zelllinie besteht aus Zellen, die durch Teilung auseinander hervorgehen und die in vitro immer weiter vermehrt werden können. Ein Beispiel für eine sehr gebräuchliche Zelllinie sind sogenannte HeLa-Zellen, die aus Tumorgewebe der Patientin Henrietta Lacks gewonnen wurden. 
genetische Auffälligkeiten auf. Zum anderen repräsentieren sie nicht die Heterogenität der Tumormasse, da jeweils nur die Zellen selektiert werden, welche sich gut in vitro vermehren lassen. Darüber hinaus werden in einschichtigen Zellkulturen viele Funktionen des weiblichen Reproduktionstraktes nicht nachgebildet und können somit auch nicht erforscht werden. Der Einsatz von Organoiden kann diese Probleme zum Teil lösen: sie können langfristig am Leben erhalten und vermehrt werden, sie bestehen aus mehreren Zelltypen, die sich selbst dreidimensional organisieren und sie besitzen funktionelle Fähigkeiten, die denen des Ursprungsorgans entsprechen.

Abhängig von bestimmten Wachstumsfaktoren und Hormonen können derzeit (Stand April 2020) bei Menschen aus einigen, bei Mäusen aus allen Bereichen des weiblichen Reproduktionstraktes Organoide gezüchtet werden. Diese Organoidkulturen bestehen aus den Epithelzellen des jeweiligen Organs. Für die Entwicklung menschlicher Eierstock-Organoide wird gegenwärtig noch nach optimalen Kulturbedingungen geforscht, da sie sich bisher nur langsam vermehren. Eileiter-Organoide können aus primären Epithelzellen des menschlichen Eileiters kultiviert werden. Die Innenschicht des Uterus, die Gebärmutterschleimhaut (Endometrium), kann bereits mit einer Erfolgsrate von fast $100 \%$ aus menschlichen Zellen gezüchtet werden. Möglich ist dies von Gewebeproben aus allen Stadien des Menstruationszyklus - auch von menopausalen und schwangeren Gewebespenderinnen. Ähnlich der Gebärmutterschleimhaut reagieren auch Endometrium-Organoide auf Hormone. Darüber hinaus gibt es Gebärmutterhals-Organoide und vaginale Organoide; Letztere allerdings bislang nur im Mausmodell.

\subsubsection{Organoide als Werkzeuge zur Untersuchung von Krankheiten des weiblichen Reproduktionstraktes}

Organoidsysteme können verschiedene Krankheiten des weiblichen Reproduktionstraktes nachbilden. Dazu gehören Endometriosen, Gebärmutterschleimhautkrebs, Eierstockkrebs, Gebärmutterhalskrebs und Infektionskrankheiten. Endometriosen treten bei ungefähr $10 \%$ der Frauen im fortpflanzungsfähigen Alter auf. Sie führen oft zu Schmerzen und chronischen Entzündungen und sind verbunden mit einer Anfälligkeit für Unfruchtbarkeit sowie für Brust- und Eierstockkrebs. Der Krankheitsverlauf wird in vier Stadien von I (mild) bis IV (schwer) unterteilt. Es wurde bereits eine Biobank mit Organoiden von Endometrioseherden aus verschiedenen klinischen Stadien eingerichtet. Die Organoide behielten auch in Kultur viele der Eigenschaften des ursprünglichen Endometrioseherdes, wie beispielsweise die Expression bestimmter Gene. Dass menschliche Organoide aus Endometrioseherden auch bei einer Injektion in Mäuse En- 
dometriose verursachen, zeigt ebenfalls, wie stark die Organoide die Eigenschaften des kranken Gewebes beibehalten. Als neues, sehr wirklichkeitsgetreues in vitro Modell der Endometriose erlauben die Organoide nicht nur die Erforschung der Krankheitsentstehung, sondern können auch zur Erforschung möglicher medikamentöser Behandlungen genutzt werden.

Gebärmutterschleimhautkrebs (Endometriumkarzinom) ist die häufigste gynäkologische Krebserkrankung. Es wurden bereits Organoide aus verschiedenen Stadien der Krebsentstehung gewonnen. Sie bilden den zellulären Aufbau sowie genomische und transkriptomische ${ }^{40}$ Eigenschaften des Tumors nach, aus dem sie gewonnen wurden. Bislang wurde zur Behandlung der betroffenen Patientinnen Strahlen- oder Chemotherapie eingesetzt, die sich gegen sich teilende Zellen richten. Nun gibt es Hoffnung, individuelle Tumore über ihre spezifischen Eigenschaften oder genetischen Programme angreifen zu können. Um hierfür geeignete Wirkstoffe zu identifizieren, können Medikamentenscreenings in kleinen Biobanken mit aus Endometriumkarzinomen hergestellten Organoiden getestet werden. So wurden beispielsweise Organoidlinien zusammen mit verschiedenen Chemotherapeutika kultiviert, woraufhin die Organoidlinien unterschiedliche Reaktionen zeigten. Dieses Vorgehen könnte auch für die personalisierte Medizin im Bereich der Krebsforschung, insbesondere der Medikamentenentwicklung bedeutsam werden (zu Organoiden in der Krebsforschung siehe Kretzschmar, Kap. 3.4).

Eierstockkrebs (Ovarialkarzinom) ist nach dem Endometriumkarzinom der zweithäufigste Genitaltumor der Frau. Die Erkrankung ist mit einer erhöhten Sterblichkeit verbunden, da sie häufig erst in einem fortgeschrittenen Stadium erkannt wird. Fünf Jahre nach der Diagnose liegt die Überlebensrate bei Patientinnen mit fortgeschrittenem Tumorstadium nur bei $25 \%$. Eierstockkrebs wird in mehrere Tumorsubtypen unterteilt, welche ein breites Spektrum verschiedener genetischer und pathologischer Charakteristika aufweisen. Es konnten bereits verschiedene Typen von Eierstockkrebs als Organoide gezüchtet und für Medikamentensensitivitätstests genutzt werden.

Gebärmutterhalskrebs (Zervixkarzinom) führt jährlich zu 300.000 Todesfällen weltweit. Dies verdeutlicht die Notwendigkeit einer Früherkennung. Tumor-Organoide aus Biopsien von Gebärmutterhalskrebs erlauben es, die Krebsentstehung zu untersuchen sowie therapeutische Ziele zu identifizieren.

Infektionen des weiblichen Reproduktionstraktes können auftreten, wenn die üblichen symbiotischen Mikroorganismen von krankheitserregenden (pathogenen) Mi-

40 Genomische und transkriptomische Eigenschaften beziehen sich auf die vorhandenen Gene sowie das Muster der Genexpression im Tumorgewebe. 
kroorganismen verdrängt werden. Diese können dann in die oberen Reproduktionsorgane einwandern. Die wichtigsten Pathogene des weiblichen Reproduktionstraktes sind Treponema pallidium (Syphilis), Neisseria gonorrhoeae, Chlamydia trachomatis, Trichomonas vaginalis, das humane Papillomavirus (HPV) und Herpes Simplex Virus. Infektion mit HPV kann zu Zervixkarzinom führen, und eine gleichzeitige Infektion mit Chlamydia trachomatis steht im Verdacht, die Krebsentstehung zu beschleunigen. Bisher fehlen jedoch adäquate humane Modelle, um diese Infektionen und die Krebsentstehung zu untersuchen. Es wurde aber kürzlich gezeigt, dass Chlamydien Organoide infizieren können und sich in den Zellen vermehren können. Es besteht daher die Hoffnung, dass sie als neues Modell auch von Ko-Infektionen zum besseren Verständnis der Interaktionen zwischen Wirt und Pathogen und der Krebsentstehung beitragen können (siehe Abbildung 2).

Abbildung 2: Zukünftige Anwendungen von Organoiden des weiblichen Reproduktionstraktes

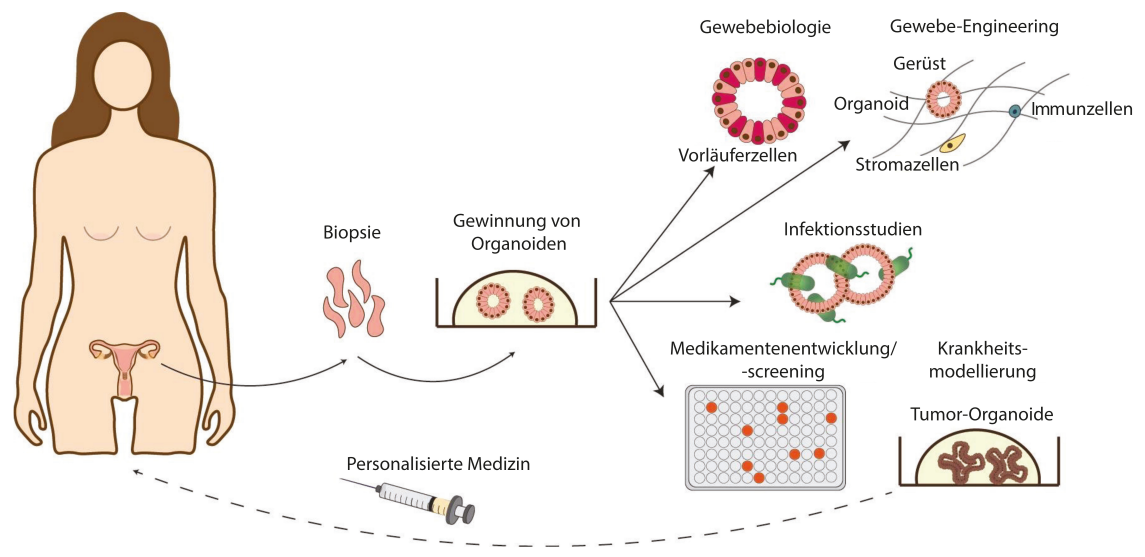

\subsubsection{Zukünftige Entwicklungen und Anwendungspotenziale}

Die sich abzeichnenden Entwicklungen in der Forschung an Organoiden des weiblichen Reproduktionstraktes sind vielfältig und spannend. Mögliche Anwendungen der Organoidtechnologie betreffen:

(i) den Einsatz von Genome-Editing, d. h. die Modifikation von Organoiden mit Genscheren (etwa CRISPR/Cas9), die es unter anderem ermöglichen, die Auswirkungen des Ausschaltens einzelner Gene auf die Entwicklung der Organoide zu untersuchen; 
(ii) die Nutzung für die personalisierte Medizin, etwa über die Generierung patientenspezifischer Organoide, an denen vorab getestet werden kann, welches Medikament bei der jeweiligen Patientin wirksam sein könnte;

(iii) das Bioengineering (siehe Teriyapirom/Batista-Rocha/Koo, Kap. 3.3), also die Kultivierung von Organoiden zusammen mit weiteren Zelltypen wie Blutgefäßen, Nervenzellen, Stromazellen und umgebender extrazellulärer Matrix; sowie

(iv) Organoide zur Untersuchung der frühen Schwangerschaft, etwa auch mittels Plazenta-Organoiden. Es könnte auch die Untersuchung einer Implantation in der Petrischale (,implantation in a dish“) möglich werden, indem man Organoide mit natürlichen oder synthetischen Embryonen gemeinsam kultiviert.

Diese neuen Ansätze werden das Forschungsfeld weiter voranbringen und Einschränkungen in der Nutzung aktueller Organoidsysteme überwinden. Während neue Technologien so die Grenzen dessen, was im Labor machbar ist, immer weiter verschieben, ist es jedoch erforderlich, auch die ethischen Fragen zu betrachten, die mit der Nutzung von Organoiden verbunden sein können (siehe Schicktanz, Kap. 6, und Nicolas/Etoc/Brivanlou, Kap. 5).

\subsubsection{Fazit}

Der weibliche Reproduktionstrakt durchläuft während des Lebens einer Frau dramatische Veränderungen. Seine Funktionsfähigkeit ist dabei essenziell für die reproduktive Gesundheit und das Wohlbefinden der Frau. Die Forschung auf diesem Gebiet wurde lange durch das Fehlen physiologisch bedeutsamer Modelle erschwert. Die beschriebene Entwicklung von Organoiden des weiblichen Reproduktionstraktes kann sowohl gesundes als auch krankes Gewebe sowie deren zentrale anatomische, molekulare und funktionelle Eigenschaften nachbilden. Die Erforschung des weiblichen Reproduktionstraktes wird durch alle beschriebenen Entwicklungen nicht nur transformiert, sondern auch entscheidend vorangebracht. 